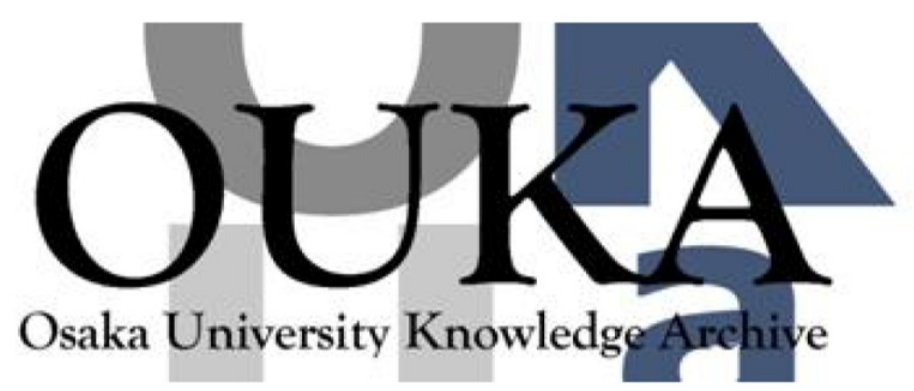

\begin{tabular}{|c|l|}
\hline Title & Minimal dilaton model \\
\hline Author(s) & $\begin{array}{l}\text { Abe, Tomohiro; Kitano, Ryuichiro; Konishi, } \\
\text { Yasufumi et al. }\end{array}$ \\
\hline Citation & Physical Review D. 86(11) p. 115016 \\
\hline Issue Date & $2012-12-07$ \\
\hline oaire:version & VoR \\
\hline URL & https://hdl. handle. net/11094/78754 \\
\hline rights & ○ 2012 American Physical Society \\
\hline Note & \\
\hline
\end{tabular}

Osaka University Knowledge Archive : OUKA

https://ir. Library. osaka-u. ac. jp/

Osaka University 


\title{
Minimal dilaton model
}

\author{
Tomohiro Abe, ${ }^{1, *}$ Ryuichiro Kitano, ${ }^{2, \dagger}$ Yasufumi Konishi, ${ }^{3, \ddagger}$ Kin-ya Oda, ${ }^{4, \S}$ Joe Sato, ${ }^{3, \|}$ and Shohei Sugiyama ${ }^{5, \pi}$ \\ ${ }^{1}$ Institute of Modern Physics and Center for High Energy Physics, Tsinghua University, Beijing 100084, China \\ ${ }^{2}$ Department of Physics, Tohoku University, Sendai 980-8578, Japan \\ ${ }^{3}$ Department of Physics, Saitama University, Saitama 355-8570, Japan \\ ${ }^{4}$ Department of Physics, Kyoto University, Kyoto 606-8502, Japan \\ ${ }^{5}$ Institute for Cosmic Ray Research (ICRR), University of Tokyo, Kashiwa, Chiba 277-8582, Japan
}

(Received 8 October 2012; published 7 December 2012)

\begin{abstract}
We construct a minimal calculable model of a light dilaton based on the scenario where only top and Higgs sectors are involved in a quasiconformal dynamics. The model consistently accommodates the electroweak precision tests even when the Higgs boson is very heavy, thereby allowing one to consider the possibility that the particle at around $125 \mathrm{GeV}$, discovered at the LHC experiments, is identified as the light dilaton rather than the Higgs boson. We find that the current LHC data allow distinct parameter regions where the observed particle is either mostly the Higgs boson or the dilaton.
\end{abstract}

DOI: 10.1103/PhysRevD.86.115016

PACS numbers: $12.60 . \mathrm{Fr}$

\section{INTRODUCTION}

It has been reported recently that both the ATLAS and the CMS experiments observed resonances in the $\gamma \gamma, Z Z$, and $W W$ channels at around $125 \mathrm{GeV}[1,2]$. The resonance can naturally be interpreted as a signal of the Higgs boson in the Standard Model (SM). The mass range suggests that the Higgs sector of the SM is fairly weakly coupled, perfectly consistent with the precision measurements at the LEP experiments.

The observation of the light Higgs boson excludes various models of electroweak symmetry breaking via strong dynamics. For example, typical technicolor models [3] and their proposed effective descriptions such as the Higgsless model [4] are in trouble with the weakly coupled descriptions of the Higgs sector.

There is, however, a logical possibility that the excess is not due to the Higgs boson but rather a Nambu-Goldstone boson associated with an approximate scale invariance in the dynamical sector. In Ref. [5], it has been proposed that the dilaton in the walking technicolor [6] may explain the signals; see also Ref. [7] for an effective theory description of dilaton systems. (There is an attempt to identify a dilaton as the Higgs [8].) In Ref. [9], the radion in the Randall-Sundrum model has been discussed as a possible candidate, see also Ref. [10].

It has been reported that the excess of events at around 126.5 (125) $\mathrm{GeV}$ at ATLAS (CMS) in the diphoton final state is enhanced from the expectation of the SM by a factor $1.8 \pm 0.5(1.6 \pm 0.4)[1,2]$. Though this tendency is

\footnotetext{
*tomohiro_abe@tsinghua.edu.cn

†kitano@tuhep.phys.tohoku.ac.jp

${ }^{\ddagger}$ konishi@krishna.th.phy.saitama-u.ac.jp

\$odakin@gauge.scphys.kyoto-u.ac.jp

॥joe@phy.saitama-u.ac.jp

"shohei@icrr.u-tokyo.ac.jp
}

not to the level of significance, it is noteworthy that the above stated models can naturally account for the trend.

If the excesses at around $125 \mathrm{GeV}$ are due to such a nonHiggs particle, one needs to consider a consistency with the constraints on the Peskin-Takeuchi $S$ and $T$ parameters [11]. This non-Higgs scenario requires the real Higgs boson (if it exists) to be heavier than $\sim 600 \mathrm{GeV}$ in order to be consistent with the LHC data, and such a heavy Higgs boson does not give a good fit to the precision measurements. It is required to have some other contributions to the $S$ and $T$ parameters. Since the models based on (unknown) technicolor theories or an extra dimension have no predictability or only weak predictability on the $S$ and $T$ parameters, it is difficult to judge if such models are really viable.

In this paper, we construct an effective (minimal) model of such a framework and see if there is a viable parameter region. The model consists of a massive vectorlike toppartner fermion. The top-partner mass $M$ represents a mass gap in the dynamical sector, to which the dilaton naturally couples in order to recover the scale invariance: $M \rightarrow M e^{-\phi / f}$. The coupling in turn provides interactions between the dilaton $\phi$ and the photons/gluons through loop diagrams of the top partners, explaining the excesses at the ATLAS and CMS. The top partner can also contribute to the Peskin-Takeuchi $S, T$ parameters, which turns out to be a good direction to come back to the allowed region, canceling the heavy SM Higgs boson contribution to the $T$ parameter.

We discuss whether such a dilaton explanation of the excesses is viable and how such a scenario can be discriminated by measuring various cross sections and decay branching ratios at the LHC experiments. The minimal model we study below catches essential features of dilaton/radion models, and the model parameters we discuss can easily be translated into those in other models.

In Sec. II, we present the minimal dilaton model as an effective renormalizable theory equipped with a linearized 
dilaton field $S$ and the vectorlike top partner $T$ [12]. In Sec. III, we show signal strengths for all the Higgs decay final states and give constraints on model parameters, namely a Higgs-dilaton mixing angle $\theta_{H}$ and a dilaton decay constant $f$, which is nothing but a vacuum expectation value of $S$. In Sec. IV, constraints from the electroweak precision measurements are examined on the top-partner sector, namely on the left-handed mixing $\theta_{L}$ between the top and its partner $T$ and on the heavier $t^{\prime}$ quark mass. The last section is devoted to summary and discussions.

\section{THE MINIMAL DILATON MODEL}

The dilaton field, defined as the Nambu-Goldstone particle associated with an approximate scale invariance of the theory, can be produced through the gluon fusion process at the LHC and can decay into two photons through the following effective operators:

$$
\phi G_{\mu \nu}^{a} G^{a \mu \nu}, \quad \phi F_{\mu \nu} F^{\mu \nu},
$$

where $\phi, G_{\mu \nu}^{a}$, and $F_{\mu \nu}$ are the dilaton, the gluon field strength, and the photon field strength, respectively. These effective operators are generated if there is a colored and charged field which obtains mass through the spontaneous breaking of the approximate scale invariance. For example, in an approximately scale invariant technicolor theory, one can expect such operators to appear at low energy if there are colored and charged techniquarks. Also, there can be a dual hadronic description of such a theory where the approximate scale invariance is nonlinearly realized. An example is a model with a warped extra dimension, where $\phi$ represents the radius of the extra dimension. In either example, predictions to the effective couplings and also constraints from the electroweak precision measurements are pretty model dependent, and moreover, there are often technical difficulties in the estimations due to large nonperturbative effects or incalculable corrections from the cutoff scale physics.

We, therefore, consider an effective minimal model of the dilaton, based on a weakly coupled renormalizable theory. The model allows us to perform explicit computations of the Peskin-Takeuchi $S, T$ parameters and also production/decay processes of the dilaton at the LHC. This exercise not only provides us with a sense of how such a model is constrained, but is also practically useful since the obtained allowed range of parameters can easily be translated into other calculable models.

The operators in Eq. (1) are obtained by integrating out a field which is colored and charged. We choose the field to have the same quantum numbers as the right-handed top quark. This is somewhat a natural choice. When we consider the origin of the large top-quark mass, one may need to assume that the top quark is (semi) strongly coupled to a dynamical sector, such as in the topcolor [13] or the top seesaw models [14]. It is then reasonable to assume an existence of a resonance with the same quantum number as the top quarks. The resonance can decay into a bottom quark and a $W$ boson, and thus does not have a problem with a exotic stable state. As a minimal choice, we consider a vectorlike top partner with the same gauge quantum number as the right-handed $S U(2)_{L}$ singlet top quark rather than the left-handed doublet that also includes bottom quark partner.

We write down the following Lagrangian for the dilaton system:

$$
\begin{aligned}
\mathcal{L}= & \mathcal{L}_{\mathrm{SM}}-\frac{e^{-2 \phi / f}}{2} \partial_{\mu} \phi \partial^{\mu} \phi-\bar{T}\left(\not D+M e^{-\phi / f}\right) T \\
& -\left[y^{\prime} \bar{T}_{R}\left(q_{3 L} \cdot H\right)+\text { h.c. }\right]-V(\phi, H),
\end{aligned}
$$

where $T$ is the heavy vectorlike top partner representing the resonance, $q_{3 L}$ is the left-handed (top and bottom) quark doublet, and $H$ is the SM Higgs doublet field. The Lagrangian $\mathcal{L}_{\mathrm{SM}}$ is the Standard Model, and the term with a coupling constant $y^{\prime}$ provides a mixing between the top quark and $T$. The Lagrangian has a nonlinearly realized scale invariance except for the scalar potential term $V(\phi, H)$ which contains terms with small explicit breaking of the scale invariance. The potential terms provide mass terms for $\phi$ as well as a mixing between $\phi$ and the Higgs boson. We choose the origin of the field $\phi$ so that $\langle\phi\rangle=0$. A mass term of $\bar{u}_{3 R} T_{L}$, with $u_{3 R}$ being the righthanded top quark, can be eliminated by an appropriate field redefinition; see Appendix A. It may be interesting to consider this model as the low-energy effective theory of the top condensation model [15], where the coupling $y^{\prime}$ and the quartic coupling constant of the Higgs field blows up simultaneously at a high-energy scale. We do not impose such a constraint in this paper in order to leave the discussion general.

\section{A. Linearized model}

By a field redefinition,

$$
S=f e^{-\phi / f},
$$

the Lagrangian given in Eq. (2) is equivalent to

$$
\begin{aligned}
\mathcal{L}= & \mathcal{L}_{\mathrm{SM}}-\frac{1}{2} \partial_{\mu} S \partial^{\mu} S-\bar{T}\left(\not D+\frac{M}{f} S\right) T \\
& -\left[y^{\prime} \bar{T}\left(q_{3 L} \cdot H\right)+\text { H.c. }\right]-\tilde{V}(S, H) .
\end{aligned}
$$

The scale invariance is now linearly realized. The potential $\tilde{V}(S, H)$ should be arranged so that $\langle S\rangle=f$ and $\left\langle H^{0}\right\rangle=$ $v / \sqrt{2}$. The explicit form of $\tilde{V}$ is shown in Appendix B for completeness, though we do not need to specify it for the following analysis as we will be discussing with physical quantities such as the masses and mixings. We propose this Lagrangian as a minimal effective description of an approximately scale invariant theory of (dynamical) electroweak symmetry breaking involving the only top and Higgs sectors.

If the mixing between the dilaton and the Higgs boson is small and if the dilaton is the one which explains the 
observed resonance at the LHC, the Higgs boson must be heavier than about $600 \mathrm{GeV}$ in order to be consistent with the Higgs boson searches at the LHC. With such a heavy Higgs boson, constraints from the Peskin-Takeuchi $S, T$ parameters require a new contribution to come back to the ellipse in the $S-T$ plane. As we will see shortly, such a contribution is already there in this model since the loop diagrams of the $t^{\prime}$ field can push $S$ and $T$ parameters towards the right direction. This Lagrangian, therefore, provides a compact realistic model of the light dilaton, which can be used for LHC studies.

\section{B. Model parameters}

This model has new parameters in addition to the Standard Model ones. We here list them and their definitions:

(i) $f$

This is the decay constant of the dilaton. The size of $f$ controls the strength of the coupling of the dilaton $\phi$ to photons and gluons and to all the fields involved in the quasiconformal dynamics. For later use, we define a dimensionless quantity,

$$
\eta=\frac{v}{f} N_{T}
$$

where $v=\sqrt{2}\left\langle H^{0}\right\rangle=246 \mathrm{GeV}$, and $N_{T}$ is the number of the $T$ fields. This parameter appears when we discuss the production and decay of $\phi$. If necessary, one can obtain a large value of $\eta$ with a large number of $N_{T}$ without requiring too small $f$. For the minimal model $N_{T}=1$ which we will assume hereafter.

(ii) $m_{s}, m_{h}$, and $\theta_{H}$

These are masses $\left(m_{s}<m_{h}\right)$ and mixing of the scalar fields. We take lighter mass eigenstate to explain the LHC excesses, i.e.,

$$
m_{s} \simeq 125 \mathrm{GeV} \text {. }
$$

The Higgs-dilaton mixing angle is defined as

$$
\begin{aligned}
S & =f+s \cos \theta_{H}-h \sin \theta_{H}, \\
H^{0} & =\frac{v+s \sin \theta_{H}+h \cos \theta_{H}}{\sqrt{2}},
\end{aligned}
$$

where $s$ and $h$ are the lighter and the heavier mass eigenstates, respectively. We impose

$$
m_{h}>600 \mathrm{GeV}
$$

to be consistent with the data from LHC. When the mixing angle is so large that the lighter one is almost the Standard Model Higgs boson, it is not necessary to impose the above constraint for a large $f$. Since we are particularly interested in a small mixing region, we always impose the above constraint in the following analysis. (iii) $m_{t^{\prime}}$ and $\theta_{L}$

These are the $T$ mass and the left-handed mixing of the top sector. The mass matrix for the top quark and its partner,

$$
\mathcal{M}_{t}=\left[\begin{array}{cc}
y_{t} v / \sqrt{2} & y^{\prime} v / \sqrt{2} \\
0 & M
\end{array}\right],
$$

are diagonalized as

$$
\begin{aligned}
& {\left[\begin{array}{cc}
\cos \theta_{L} & -\sin \theta_{L} \\
\sin \theta_{L} & \cos \theta_{L}
\end{array}\right] \mathcal{M}_{t} \mathcal{M}_{t}^{\dagger}\left[\begin{array}{cc}
\cos \theta_{L} & \sin \theta_{L} \\
-\sin \theta_{L} & \cos \theta_{L}
\end{array}\right]} \\
& \quad=\left[\begin{array}{cc}
m_{t}^{2} & 0 \\
0 & m_{t^{\prime}}^{2}
\end{array}\right]
\end{aligned}
$$

where $m_{t}$ is the top quark mass. One can trade the new parameters $y^{\prime}$ and $M$ by the observables $m_{t^{\prime}}$ and $\theta_{L}$, while $y_{t}$ should be adjusted to reproduce the top quark mass. See Appendix A for more detailed discussion. In Sec. IV, we verify constraints on the parameters $\theta_{L}$ and $m_{t^{\prime}}$ from the electroweak precision data.

\section{Coupling to the Standard Model fields}

The loop diagrams of $T$ generate the effective couplings in Eq. (1). In the limit of $2 m_{t^{\prime}} \gg m_{s}$, the effective couplings for the production and decays of $s$ are approximated by momentum independent pieces which are insensitive to $m_{t^{\prime}}$ or $\theta_{L}$. This behavior can most easily be understood by identifying the effective coupling as the dilaton/Higgs dependence of the running gauge coupling constants:

$$
\mathcal{L}_{\text {eff }}=-\frac{1}{4 g_{s}^{2}} G_{\mu \nu}^{a} G^{a \mu \nu},
$$

where we take the gluon as an example. The running coupling constant at a low-energy scale is given by

$$
\begin{aligned}
\frac{1}{g_{s}^{2}(\mu)}= & \frac{1}{g_{s}^{2}(\Lambda)}-\frac{2\left(b_{\mathrm{SM}}+\Delta b\right)}{(4 \pi)^{2}} \log \frac{m_{t^{\prime}}}{\Lambda} \\
& -\frac{2 b_{\mathrm{SM}}}{(4 \pi)^{2}} \log \frac{m_{t}}{m_{t^{\prime}}}-\frac{2\left(b_{\mathrm{SM}}-\Delta b\right)}{(4 \pi)^{2}} \log \frac{\mu}{m_{t}},
\end{aligned}
$$

at one-loop level, where $b_{\mathrm{SM}}=-7$ and $\Delta b=2 / 3$. This can be rearranged to

$$
\begin{aligned}
\frac{1}{g_{s}^{2}(\mu)}= & \frac{1}{g_{s}^{2}(\Lambda)}-\frac{2\left(b_{\mathrm{SM}}-\Delta b\right)}{(4 \pi)^{2}} \log \frac{\mu}{\Lambda} \\
& -\frac{2 \Delta b}{(4 \pi)^{2}} \log \frac{\left(y_{t}\left\langle H^{0}\right\rangle\right)(M\langle S\rangle / f)}{\Lambda^{2}},
\end{aligned}
$$

where we have used the fact that

$$
m_{t} m_{t^{\prime}}=y_{t}\left\langle H^{0}\right\rangle \frac{M\langle S\rangle}{f},
$$

which is derived from the mass matrix in Eq. (9). By recovering the field fluctuations $\left\langle H^{0}\right\rangle,\langle S\rangle \rightarrow H^{0}, S$ and 
considering the mixing factors in Eq. (7), we obtain the effective coupling of $s$ to gluons from Eq. (11) as

$$
\mathcal{L}_{\mathrm{eff}}^{(\mathrm{sgg})}=\frac{1}{4} \frac{g_{s}^{2}}{(4 \pi)^{2}} \frac{2}{v} \frac{2}{3}\left(\eta \cos \theta_{H}+\sin \theta_{H}\right) s G_{\mu \nu}^{a} G^{a \mu \nu},
$$

where we have canonically normalized the kinetic term of the gluon. The terms proportional to $\sin \theta_{H}$ and $\eta \cos \theta_{H}$ are contributions from the SM top and its partner, respectively. We can explicitly see that the coupling is independent of $m_{t^{\prime}}$ or $\theta_{L}$. For the $s$ to photon coupling, we need to include a loop of the $W$ bosons. The result is

$\mathcal{L}_{\mathrm{eff}}^{(s \gamma \gamma)}=\frac{1}{4} \frac{e^{2}}{(4 \pi)^{2}} \frac{2}{v}\left(\frac{4}{3} N_{c} Q_{t}^{2} \eta \cos \theta_{H}+A_{\mathrm{SM}} \sin \theta_{H}\right) s F_{\mu \nu} F^{\mu \nu}$,

where $N_{c}=3$ and $Q_{t}=2 / 3$ are the color factor and the top quark charge, respectively, and the explicit form of the loop factor $A_{\mathrm{SM}} \simeq-6.5$ can be found e.g., in (2.45) in Ref. [16]. The first term in the parentheses is the contribution from the top-partner loop whereas the second is from the SM top and $W$ ones.

The particle $s$ can also couple to the $W$ and $Z$ bosons and the fermions through the $\theta_{H}$ mixing. The couplings are simply given by those of the SM Higgs boson times a factor of $\sin \theta_{H}$.

Note here that the model is not the same as the Higgsdilaton model studied in Refs. [17-19], where the coupling between the dilaton and the Standard Model fields are assumed to have the form

$$
\mathcal{L}_{\text {int }}=\frac{\phi}{f} T_{\mu}^{\mu} .
$$

Here, $T^{\mu}{ }_{\mu}$ is the trace of the energy-momentum tensor of the Standard Model. Through this term, the dilaton directly couples to the violation of the scale invariance in the Standard Model, i.e., to the $W, Z$ bosons and fermions with strength proportional to their masses. Also, the couplings to the photons and gluons are proportional to the beta functions. The effective interaction term in Eq. (17) is generated at low energy if the whole Standard Model sector is a part of the scale invariant theory in the UV; all the gauge bosons and fermions are composite particles.

In contrast, we take a more conservative picture that the Standard Model except for the top/Higgs sector is a spectator of the dynamics, and thus the dilaton couples to the $W, Z$ bosons and fermions only through the mixing with the Higgs fields. The couplings to the gluons and photons are generated only through the loops of $t$ and $t^{\prime}$. Due to these different origin of the couplings between two models, the production and decay properties are quite different. Indeed, we will see that our model can give better fit to the LHC data compared to the SM Higgs boson, while Refs. [17-19] have reported that the dilaton scenario based on Eq. (17) is rather disfavored.
In terms of the parameters in Refs. [17-19], our model corresponds to

$$
\begin{aligned}
c_{V} & =c_{F}=\sin \theta_{H}, \\
c_{t} & =\cos ^{2} \theta_{L} \sin \theta_{H}+\eta \sin ^{2} \theta_{L} \cos \theta_{H}, \\
c_{g} & =\eta \cos \theta_{H}+\sin \theta_{H}, \\
c_{\gamma} & =\eta A_{t^{\prime}} \cos \theta_{H}+A_{\mathrm{SM}} \sin \theta_{H}, \\
c_{\mathrm{inv}} & =0,
\end{aligned}
$$

where $F$ stands for all the SM fermions except the top quark. (The parameter $c_{X}$ for a production/decay process $X$ is written as $\kappa_{X}$ in a recent analysis by ATLAS [20]. Our notation in the forthcoming Eqs. (21)-(27) reads $R_{X}=c_{X}^{2}=\kappa_{X}^{2}$.) It is worth noting that a negative value for $c_{F}$ can be easily obtained in our model, which tends to be more favored than the SM value $c_{F}=1$ in order to suppress the $c_{g}$ coupling while keeping $c_{\gamma}$ large [17-19].

\section{DILATON AT THE LHC}

As we discussed in Sec. II, there are two mass eigenstates in the scalar sector, $s$ and $h$, and their couplings are determined by two parameters, $\eta$ and $\theta_{H}$ in Eqs. (5) and (7). In the small $\theta_{H}$ region, $s$ is dilatonlike as we can see from Eq. (7). In the following, we assume the lighter mass eigenstate $s$ to be around $125 \mathrm{GeV}$ and study the production and decays of $s$ at the LHC.

\section{A. Production}

As one can see from the above discussion, the $s$ particle has suppressed couplings to $W, Z$ and fermions, and either enhanced or suppressed couplings to $\gamma$ and the gluon compared to the Higgs boson in the Standard Model. The production cross section of $s$ compared to that of the SM Higgs boson (at the same mass as $s$ ) through a process $X$,

$$
R_{X}:=\frac{\sigma_{X}}{\sigma_{X}^{\mathrm{SM}}},
$$

is given by

$$
\begin{gathered}
R_{\mathrm{GF}}=\left(\eta \cos \theta_{H}+\sin \theta_{H}\right)^{2}, \\
R_{\mathrm{VBF}}=R_{\mathrm{VH}}=\sin ^{2} \theta_{H}, \\
R_{\mathrm{ttH}}=\left(\cos ^{2} \theta_{L} \sin \theta_{H}+\eta \sin ^{2} \theta_{L} \cos \theta_{H}\right)^{2},
\end{gathered}
$$

for the gluon fusion (GF), the vector boson fusion (VBF), the Higgs-strahlung $(\mathrm{VH})$, and the associated production with a $t \bar{t}$ pair $(\mathrm{ttH})$, respectively; see e.g., Ref. [16] for a review. Here and hereafter $V(V V)$ denotes either $W$ or $Z$ $(W W$ or $Z Z$ ). Note that the SM cross section in the denominator in Eq. (20) is evaluated at $m_{h} \simeq 125 \mathrm{GeV}$ for comparison to the experimental data, while its value in our model is $m_{h} \geq 600 \mathrm{GeV}$. As said above, $\eta$ appearing in 
Eq. (21) is not a ratio of the Yukawa couplings but that of the vacuum expectation values which can also be checked by a direct loop computation in the linearized model given in Eq. (4). We plot $R_{\mathrm{GF}}$ in the left panel of Fig. 1. The ttH ratio (23) reduces to $R_{\mathrm{ttH}} \rightarrow \sin ^{2} \theta_{H}$ in the small top mixing limit $\theta_{L} \ll 1$, which we will assume hereafter. The validity of this approximation will be confirmed in Sec. IV. We note that the $\mathrm{ttH}$ process gives negligible contribution to our diphoton analysis in Eq. (32), with $\varepsilon_{\mathrm{ttH}}^{i}$ being at most $4 \%$, and that we include it just for completeness.

\section{B. Decay}

For each decay process $s \rightarrow X$, we define the decay width ratio to that of the SM Higgs at $125 \mathrm{GeV}$,

$$
R(s \rightarrow X)=\frac{\Gamma_{s \rightarrow X}}{\Gamma_{h \rightarrow X}^{\mathrm{SM}}} .
$$

The minimal dilaton model predicts

$$
\begin{gathered}
R(s \rightarrow \text { others })=\sin ^{2} \theta_{H}, \\
R(s \rightarrow g g)=\left(\eta \cos \theta_{H}+\sin \theta_{H}\right)^{2}, \\
R(s \rightarrow \gamma \gamma)=\left(\eta \frac{A_{t^{\prime}}}{A_{\mathrm{SM}}} \cos \theta_{H}+\sin \theta_{H}\right)^{2},
\end{gathered}
$$

where the subscript "others" denotes the tree-level processes $b b, V V, \tau \tau, c c$, etc. and

$$
A_{t^{\prime}}:=N_{c} Q_{t^{\prime}}^{2} A_{\frac{1}{2}}\left(\frac{m_{s}^{2}}{4 m_{t^{\prime}}^{2}}\right) \simeq \frac{16}{9},
$$

with the loop function $A_{\frac{1}{2}}$ given in Eq. (2.46) in Ref. [16]. The ratio for the total decay width is

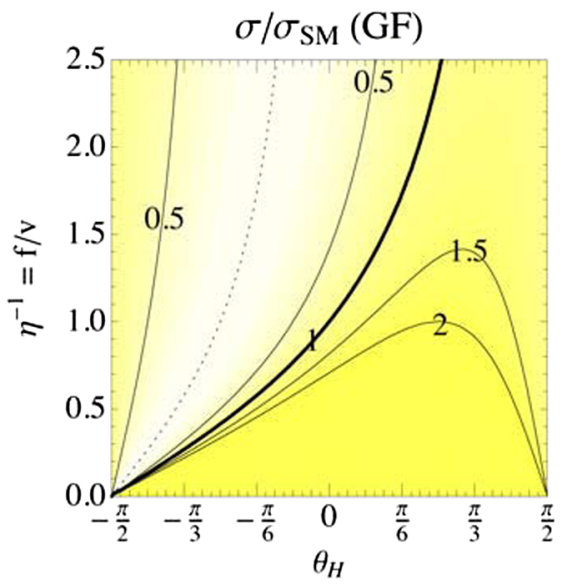

$$
\begin{aligned}
R(s \rightarrow \text { all })= & \frac{\Gamma_{s \rightarrow \text { others }}+\Gamma_{s \rightarrow g g}+\Gamma_{s \rightarrow \gamma \gamma}}{\Gamma_{h \rightarrow \text { all }}^{\mathrm{SM}}} \\
= & \mathrm{BR}_{\mathrm{others}}^{\mathrm{SM}} \sin ^{2} \theta_{H}+\mathrm{BR}_{g g}^{\mathrm{SM}}\left(\eta \cos \theta_{H}+\sin \theta_{H}\right)^{2} \\
& +\mathrm{BR}_{\gamma \gamma}^{\mathrm{SM}}\left(\eta \frac{A_{t^{\prime}}}{A_{\mathrm{SM}}} \cos \theta_{H}+\sin \theta_{H}\right)^{2}
\end{aligned}
$$

where at around $125 \mathrm{GeV}$, branching ratios in the SM are given e.g., in Ref. [17],

$\mathrm{BR}_{\text {others }}^{\mathrm{SM}}=91.3 \%, \quad \mathrm{BR}_{g g}^{\mathrm{SM}}=8.5 \%, \quad \mathrm{BR}_{\gamma \gamma}^{\mathrm{SM}}=0.2 \%$.

We plot $R(s \rightarrow$ all $)$ in the right panel of Fig. 1.

\section{Dilaton vs SM Higgs signal strengths}

Both ATLAS and CMS experiments discovered a new particle at around $125 \mathrm{GeV}$ in the diphoton [21,22], $Z Z \rightarrow 4 l[23,24]$, and $W W \rightarrow l \nu l \nu[25,26]$ channels. The obtained data for each channel are translated into the signal strength, which is an expected production cross section for a particle that decays the same as in the SM Higgs at the same mass. We constrain the model parameters $\theta_{H}$ and $\eta^{-1}=f / v$ from these three channels.

The minimal dilaton model predicts different production cross sections between GF and $\mathrm{VBF} / \mathrm{VH} / \mathrm{ttH}$ processes. In $H \rightarrow \gamma \gamma$ search, composition of these production channels differs category by category and are summarized in Table 2 in Ref. [2] for CMS and in Table 6 in Ref. [21] for ATLAS. We define $\varepsilon_{X}^{i}$ as the proportion of the production process $X$ within a category $i$. Note that $\sum_{X} \varepsilon_{X}^{i}=1$ by definition for each category $i$, where a summation over $X$ is always understood as for all the relevant production channels: GF, VBF, VH, and ttH. GF is the dominant production process and satisfies $\varepsilon_{\mathrm{GF}}^{i} \lesssim 90 \%$ in production processes other than dijet category. In the dijet category, the dominant production process is $\mathrm{VBF}$, and $\varepsilon_{\mathrm{VBF}} \lesssim 70 \%$.

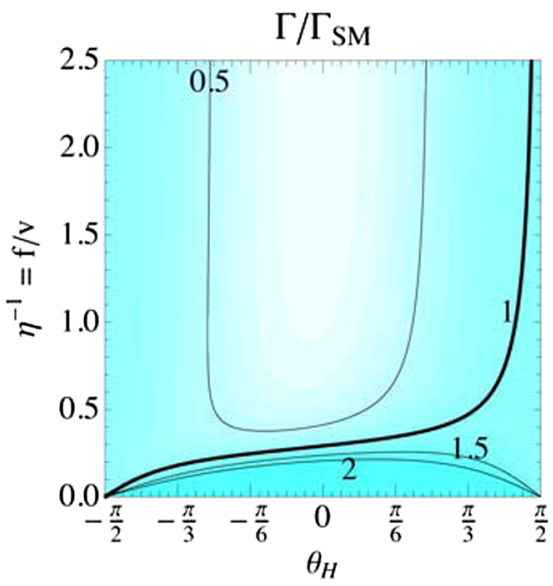

FIG. 1 (color online). Ratio to the SM for the dominant GF production cross section given in Eq. (21) (left) and to the total decay width given in Eq. (29) (right). Contours 0, 0.5, 1, 1.5, and 2 are drawn, with 0 and 1 being dotted and thick lines, respectively. A denser region gives larger value, with density changing for each increase of the ratio by 0.1 from 0 to 2 . Both sides $\theta_{H}= \pm \pi / 2$ correspond to the SM. 
When acceptance of a production channel $X$ for a category $i$ is $a_{X}^{i}$, the estimated value of a signal fraction under the given set of cuts $i$ becomes

$$
\varepsilon_{X}^{i}=\frac{a_{X}^{i} \sigma_{X}^{\mathrm{SM}}}{\sum_{Y} a_{Y}^{i} \sigma_{Y}^{\mathrm{SM}}}
$$

where $\sigma_{X}^{\mathrm{SM}}$ is the Higgs production cross section in the SM through the channel $X$. Given $\left\{\varepsilon_{X}^{i}\right\}$, we can compute the signal strength under the imposed cuts for each category $i$,

$$
\begin{aligned}
\hat{\mu}_{i}(h \rightarrow \gamma \gamma) & =\frac{\sum_{X} a_{X}^{i} \sigma_{X}}{\sum_{Y} a_{Y}^{i} \sigma_{Y}^{\mathrm{SM}}} \frac{\mathrm{BR}(s \rightarrow \gamma \gamma)}{\operatorname{BR}(h \rightarrow \gamma \gamma)_{\mathrm{SM}}} \\
& =\sum_{X} \varepsilon_{X}^{i} R_{X} \frac{R(s \rightarrow \gamma \gamma)}{R(s \rightarrow \text { all })} .
\end{aligned}
$$

We have assumed that the acceptance $a_{X}^{i}$ under the category $i$ does not change from that of the SM for each production channel $X$.

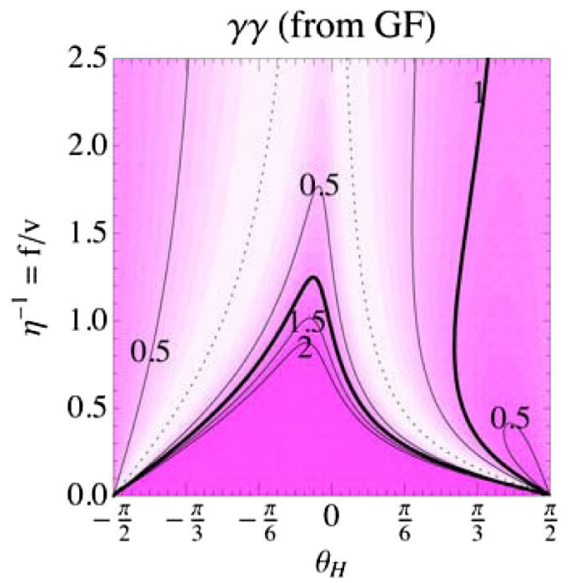

As an illustration, we plot each contribution from the initial state $X$ in the signal strength $\hat{\mu}(s \rightarrow F)$,

$$
\hat{\mu}_{X}(s \rightarrow F)=R_{X} \frac{R(s \rightarrow F)}{R(s \rightarrow \text { all })},
$$

where explicit form of all the rates in right-hand side has already been presented in Sec. III B. Figures 2 and 3 are, respectively, for the diphoton final state and for the others than diphoton and digluon ones. Though it is hardly observable at the LHC, we also plot signal the strength for digluon final states in Fig. 4 for completeness. For real experimental data under a given set of cuts $i$, signal strength becomes a mixture of those from GF and VBF, $\mathrm{VH}, \mathrm{ttH}$ processes shown in the left and right panels, respectively, with coefficients $\varepsilon_{X}^{i}(X=\mathrm{GF}, \mathrm{VBF}, \mathrm{VH}$, and $\mathrm{ttH}$ ) being multiplied as in Eq. (32). Figures $2-4$ are the prediction of our model.

From Fig. 2, we see that the diphoton signal strength can be larger than unity when the dilaton decay constant $f$ is not much larger than the SM Higgs vacuum expectation

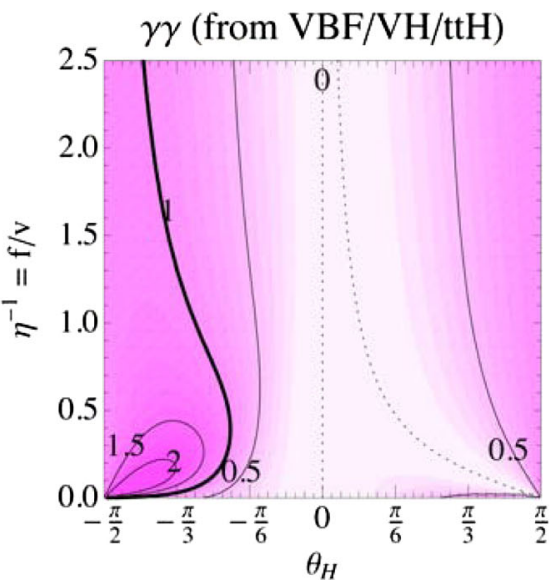

FIG. 2 (color online). Diphoton $s \rightarrow \gamma \gamma$ signal strength $\hat{\mu}$ when the production is purely from the GF (VBF/VH/ttH) process in the left (right) panel. Drawn the same as in Fig. 1.
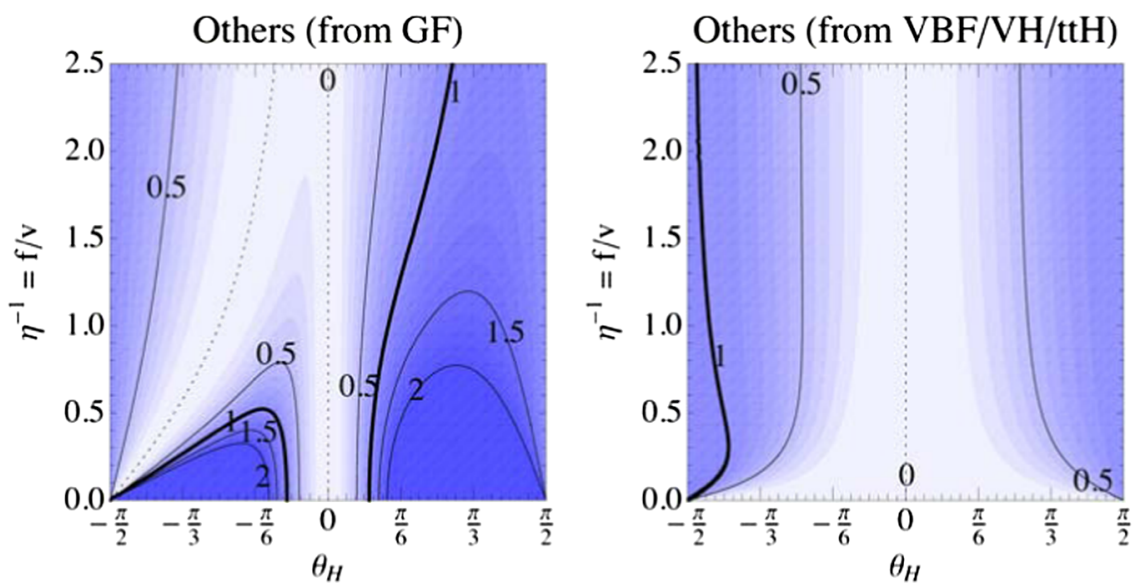

FIG. 3 (color online). Signal strength $\hat{\mu}$ for processes other than diphoton and digluon, namely for final states $s \rightarrow Z Z, W W, \tau \tau, b b$, etc. Drawn the same as in Fig. 2. 
value (VEV), namely when $\eta^{-1}=f / v \leqq 1$. In a pure $\mathrm{VBF} / \mathrm{VH} / \mathrm{ttH}$ production channel, only the negative $\theta_{H}$ region can give an enhancement of the diphoton signal strength. We can see from the right panels in Figs. 2-4 that in purely dilatonic region $\theta_{H} \simeq 0$, the $\mathrm{VBF} / \mathrm{VH} / \mathrm{ttH}$ production is suppressed for all the decay modes. In particular, the other decay modes $W W, Z Z, b b, \tau \tau$, etc. are always suppressed in the $\mathrm{VBF} / \mathrm{VH} / \mathrm{ttH}$ channel. The signal strengths for decay modes other than $\gamma \gamma$ are generally enhanced with GF production for a positive $\theta_{H}$, as can be seen in left panels of Figs. 3 and 4. Especially the digluon signal strength can be enhanced as large as 30 .

\section{Constraints on dilaton/Higgs sector}

As all the signal strengths are obtained, we perform a chi-square test with the Gaussian approximation for all the errors,

$$
\chi^{2}=\sum_{i}\left(\frac{\hat{\mu}_{i}-\mu_{i}}{\sigma_{i}}\right)^{2}
$$

where summation over $i$ is for all the diphoton categories as well as the $W W$ and $Z Z$ channels. For the $Z Z \rightarrow 4 l$ and $W W \rightarrow l \nu l \nu$ decay channels, we assume that all the signals are coming from GF and, hence, we approximate

$$
\hat{\mu}(s \rightarrow V V)=R_{\mathrm{GF}} \frac{R(s \rightarrow \text { others })}{R(s \rightarrow \text { all })}
$$

for $V V=W W$ and $Z Z$. For ATLAS, the central value $\mu_{i}$ and deviation $\sigma_{i}$ are read off from Fig. 14 in Ref. [21] for diphoton channels and from Fig. 10 in Ref. [1] for $W W$ and ZZ channels. For CMS, Fig. 6(b) in Ref. [22] and Table 7 in Ref. [2] are used for diphoton and $V V$ channels, respectively. The resultant number of degrees of freedom is 22 and 13 for ATLAS and CMS, respectively.
The results are shown in Fig. 5. We see that both experiments have allowed dilatonlike region $\left|\theta_{H}\right|<\pi / 4$ within 90\% confidence interval though the ATLAS disfavors the purely dilatonic case $\theta_{H} \simeq 0$ outside the $95 \%$ confidence interval. This is one of our main results.

As an illustration, we have also presented in right panel of Fig. 5 a "theorist combination" plot with the data from Fig. 3 in Ref. [18]. We have assumed that $W W, Z Z$, and $\gamma \gamma$ $(b b V, W W V$ and $\tau \tau)$ are all coming from GF (VBF/VH/ $\mathrm{ttH})$ processes whereas $\gamma \gamma j j$ has $70 \%$ from $\mathrm{VBF} / \mathrm{VH} / \mathrm{ttH}$ and $30 \%$ from GF. In this naive treatment, we see that the SM is already outside the $90 \%$ confidence interval whereas the minimal dilaton model has the allowed regions with a dilatonlike scalar.

\section{CONSTRAINTS ON TOP SECTOR FROM ELECTROWEAK DATA}

When the Higgs-dilaton mixing $\theta_{H}$ is small, the relevant parameters for the Peskin-Takeuchi $S, T$ parameters are the top-partner mass $m_{t^{\prime}}$ and the left-handed top mixing $\theta_{L}$. As we will see later, physics of the dilaton at the LHC is independent of those two parameters $m_{t^{\prime}}, \theta_{L}$ in the top sector. Therefore, one can discuss the electroweak constraints and the LHC physics independently. In this section, we present allowed region of the parameters $m_{t^{\prime}}$ and $\theta_{L}$ from the electroweak precision measurements.

As is well known, the electroweak precision tests prefer a light Higgs boson in the Standard Model. The upper bound is $185 \mathrm{GeV}$ at the $95 \%$ confidence level (CL) [27]. On the other hand, the assumption that the $125 \mathrm{GeV}$ excesses at the LHC as the dilaton requires the SM Higgs boson (if exists) to be heavier than about $600 \mathrm{GeV}$. It is then necessary that the $t^{\prime}$ loops provide a correction with an appropriate size and sign to push back to the allowed region in the $S-T$ plane.
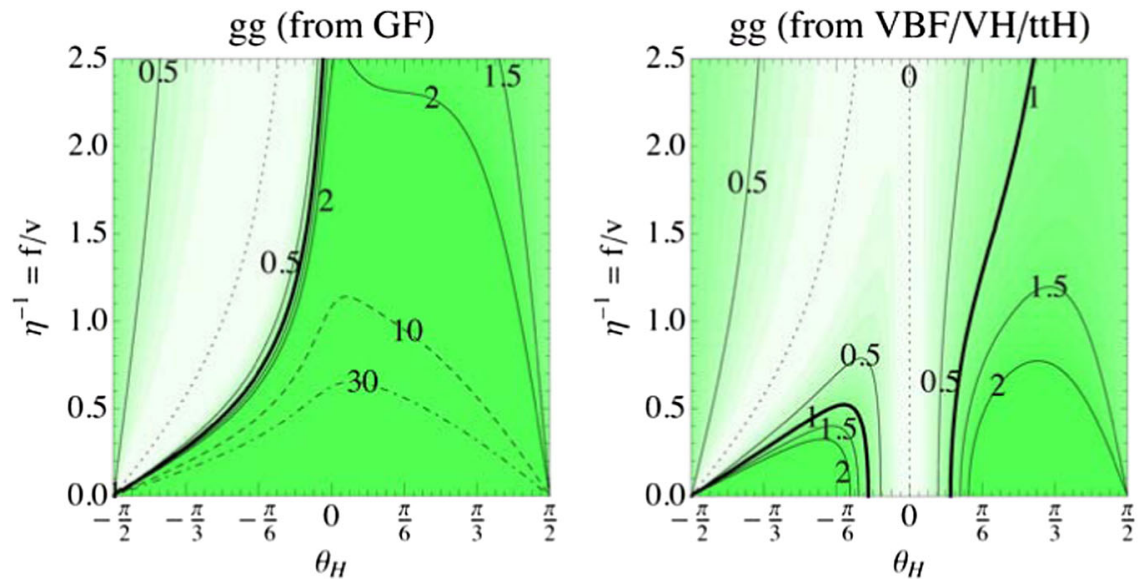

FIG. 4 (color online). Signal strength $\hat{\mu}$ for the digluon $s \rightarrow g g$ process. Drawn the same as in Fig. 2. Dashed and dot-dashed contours are added for $\hat{\mu}=10$ and 30 , respectively, in the left panel. 

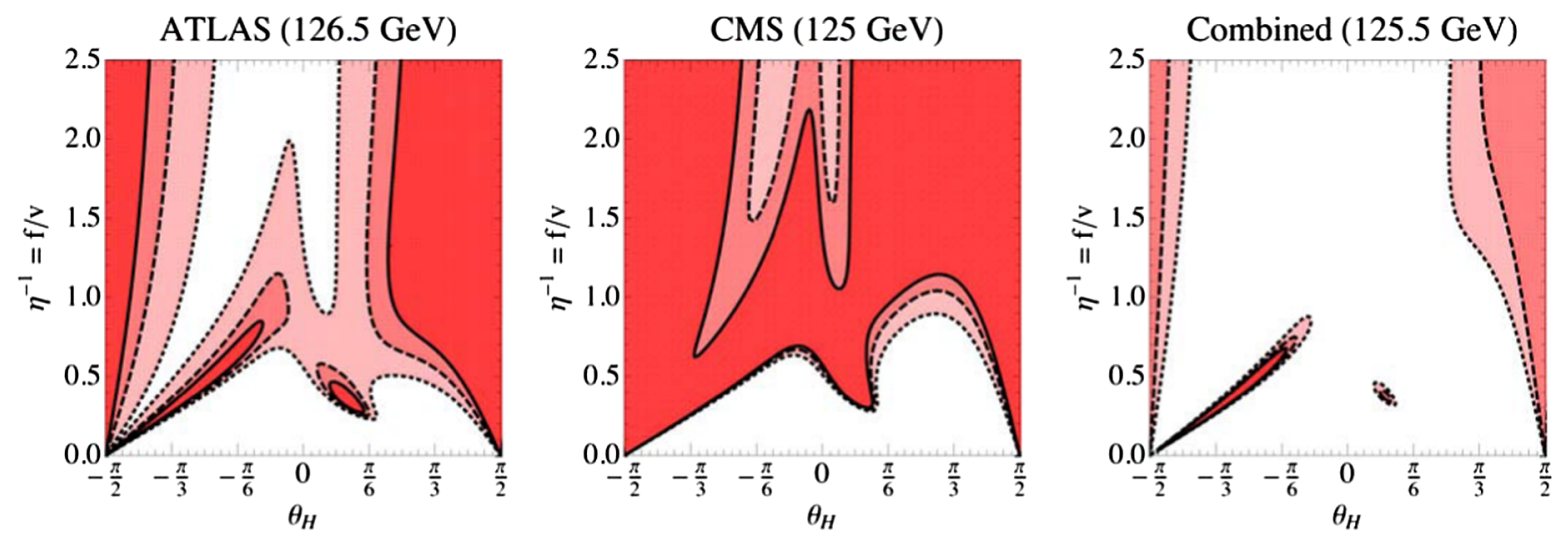

FIG. 5 (color online). Favored regions within 90, 95, and 99\% confidence intervals, enclosed by solid, dashed, and dotted lines, respectively. Density (area) of favored region decreases (increases) in according order. Results are shown for ATLAS (left), CMS (center), and combined (right). See text for details.

We have obtained contributions to the Peskin-Takeuchi $S, T$ parameters from the $t$ and $t^{\prime}$ loops as

$$
\begin{aligned}
& S^{\mathrm{top}}= \sin ^{2} \theta_{L} \frac{N_{c}}{6 \pi}\left[\left(\frac{1}{3}-\cos ^{2} \theta_{L}\right) \ln x\right. \\
&\left.+\left(\frac{(1+x)^{2}}{(1-x)^{2}}+\frac{2 x^{2}(3-x)}{(1-x)^{3}} \ln x-\frac{8}{3}\right) \cos ^{2} \theta_{L}\right], \\
& T^{\mathrm{top}}=\sin ^{2} \theta_{L} \frac{N_{c}}{16 \pi} \frac{1}{s_{W}^{2} c_{W}^{2}} \frac{m_{t}^{2}}{m_{Z}^{2}}\left[\frac{\sin ^{2} \theta_{L}}{x}\right. \\
&\left.\quad-\left(1+\cos ^{2} \theta_{L}\right)-\frac{2}{1-x} \cos ^{2} \theta_{L} \ln x\right],
\end{aligned}
$$

where

$$
x:=\frac{m_{t}^{2}}{m_{t^{\prime}}^{2}}<1
$$

and $\theta_{L}$ is the mixing angle between $t$ and $t^{\prime}$ defined in Appendix A. If $\theta_{L}=0$, then $t^{\prime}$ decouples, and $S^{\text {top }}$ and $T^{\text {top }}$ become 0 . This is because we have already subtracted the SM contributions from the definition of $S$ and $T$ parameters, as usual. For a fixed $\theta_{L}$ and a large $m_{t^{\prime}}, T^{\text {top }}$ is enhanced as $\propto m_{t^{\prime}}^{2}$, whereas $S^{\text {top }}$ only has a logarithmic dependence. Therefore, we generically obtain a large positive contribution to $T^{\text {top }}$ and $\left|S^{\text {top }}\right| \ll T^{\text {top }}$. Interestingly, this is indeed the required direction to come back to the allowed region when the Higgs boson is heavy.

We also need to calculate the contributions from scalar sectors because now we have two scalars, $s$ and $h$, and their couplings to the gauge boson are different from the SM Higgs couplings. We find

$$
\begin{aligned}
S^{\text {scalar }}= & -\frac{\cos ^{2} \theta_{H}}{12 \pi} \ln \frac{m_{\text {href }}^{2}}{m_{h}^{2}}-\frac{\sin ^{2} \theta_{H}}{12 \pi} \ln \frac{m_{\text {href }}^{2}}{m_{s}^{2}} \\
& +\cos ^{2} \theta_{H} f_{S}\left(m_{h}\right)+\sin ^{2} \theta_{H} f_{S}\left(m_{s}\right)-f_{S}\left(m_{\text {href }}\right), \\
T^{\text {scalar }}= & \frac{3 \cos ^{2} \theta_{H}}{16 \pi c_{W}^{2}} \ln \frac{m_{\text {href }}^{2}}{m_{h}^{2}}+\frac{3 \sin ^{2} \theta_{H}}{16 \pi c_{W}^{2}} \ln \frac{m_{\text {href }}^{2}}{m_{s}^{2}} \\
& +\cos ^{2} \theta_{H} f_{T}\left(m_{h}\right)+\sin ^{2} \theta_{H} f_{T}\left(m_{s}\right)-f_{T}\left(m_{\text {href }}\right),
\end{aligned}
$$

where $m_{\text {href }}$ is the reference Higgs boson mass and $f_{S}\left(m_{h}\right)$ and $f_{T}\left(m_{h}\right)$ are small nonlogarithmic contributions whose explicit expression are

$$
\begin{aligned}
f_{S}\left(m_{h}\right)= & -\frac{1}{12 \pi} \frac{\left(9 m_{h}^{2}+m_{Z}^{2}\right) m_{Z}^{4}}{\left(m_{h}^{2}-m_{Z}^{2}\right)^{3}} \ln \frac{m_{Z}^{2}}{m_{h}^{2}} \\
& -\frac{\left(2 m_{h}^{2}+3 m_{Z}^{2}\right) m_{Z}^{2}}{6 \pi\left(m_{h}^{2}-m_{Z}^{2}\right)^{2}}
\end{aligned}
$$

$$
\begin{aligned}
f_{T}\left(m_{h}\right)= & -\frac{3 m_{Z}^{2}}{16 \pi s_{W}^{2} c_{W}^{2}\left(m_{h}^{2}-m_{Z}^{2}\right)} \ln \frac{m_{h}^{2}}{m_{Z}^{2}} \\
& +\frac{3 m_{W}^{2}}{16 \pi s_{W}^{2}\left(m_{h}^{2}-m_{W}^{2}\right)} \ln \frac{m_{h}^{2}}{m_{W}^{2}} .
\end{aligned}
$$

Note that the $S$ and $T$ parameters given in Eqs. (39) and (40) are independent of the sign of the Higgs-dilaton mixing angle $\theta_{H}$ since they are functions of $\sin ^{2} \theta_{H}$.

The contributions from $S^{\text {scalar }}$ and $T^{\text {scalar }}$ tend to be smaller than the contributions from the top sector. The region in which they give non-negligible contributions is around $\sin ^{2} \theta_{H}=1$. In this region, we can not ignore the sizable contribution from the term which is proportional to $\ln \left(m_{\text {href }}^{2} / m_{s}^{2}\right)$. However, in this region, the $s$ couplings to the SM particles become almost the same as the SM Higgs boson couplings, and $h$ behaves like SM singlet particle. Then this region is nothing but the SM limit, which is not the interest in this paper. Therefore, we can conclude that the dominant contributions to the $S$ and $T$ parameters arise 
from the top sector, and $\theta_{H}$ dependence of the $S$ and $T$ parameters is mild.

The numerical values of parameters we use are

$s_{W}^{2}=0.23, \quad v=246 \mathrm{GeV}, \quad m_{s}=125 \mathrm{GeV}$,

$m_{h}=600, \quad 1000 \mathrm{GeV}, \quad\left|\theta_{H}\right|=0, \frac{\pi}{6}, \frac{\pi}{3}$.

In Fig. 6, we show favored region in the $m_{t^{\prime}}-\sin \theta_{L}$ plane. White regions are excluded at $95 \% \mathrm{CL}$ by $S$ and $T$ parameters.

There are other experimental constraints as well as $S$ and $T$ parameters. The mass bound on $t^{\prime}$ from the direct search at the LHC is [28]

$$
m_{t^{\prime}}>560 \mathrm{GeV} \quad(95 \% \mathrm{CL}) \text {. }
$$

We can find a constraint on $\theta_{L}$ from the bound on $V_{t b}$ because the mixing angle $\theta_{L}$ changes the top quark couplings, such as $g_{W t b}$. The bound on $V_{t b}$ without assuming the unitarity triangle is [29]

$$
0.81<\left|V_{t b}\right| \leq 1 \quad \text { (95\% CL). }
$$

If we assume the top quark never mixed with light quarks, then the above constraint gives

$$
0.81<\left|\cos \theta_{L}\right| \leq 1,
$$

namely,

$$
\left|\sin \theta_{L}\right|<0.59 \text {. }
$$

We find that the constraints given in Eqs. (44) and (47) are easily satisfied in the allowed region in Fig. 6.

We also study the constraint from $Z b_{L} b_{L}$ coupling. In the SM case, the flavor-dependent corrections to this coupling are proportional to the squared top-Yukawa coupling. In this model, this correction is modified due to the mixing between $t$ and $t^{\prime}$. We parametrize $Z b_{L} \bar{b}_{L}$ coupling as follows:

$$
\frac{e}{s_{W} c_{W}}\left(-\frac{1}{2}+\delta g_{L}+\frac{1}{3} s_{W}^{2}\right) .
$$

We focus on only the flavor-dependent correction hereafter because we use the constraint on $R_{b}$ [30] to derive the constraint on $Z b_{L} b_{L}$. We find that the flavor-dependent part of $\delta g_{L}$ is given by

$$
\delta g_{L}=\frac{m_{t}^{2}}{(4 \pi)^{2} v^{2}}+\delta g_{L}^{\text {new }}
$$

where the first term is the SM contribution, and the second terms is the additional contributions due to the $t^{\prime}$ and the mixing angle $\theta_{L}$. We find
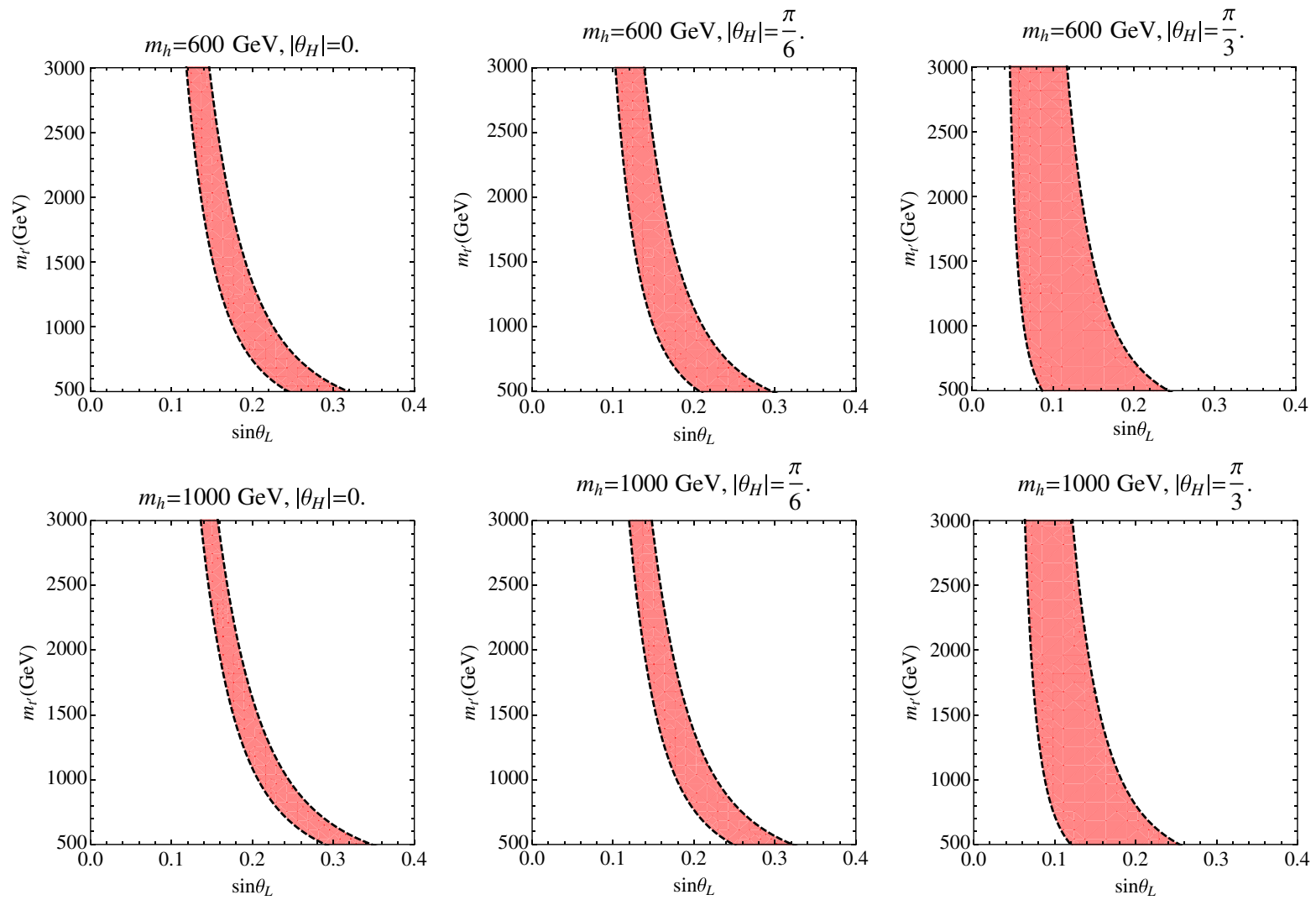

FIG. 6 (color online). Favored region plot from the Peskin-Takeuchi $S, T$. White regions are excluded at $95 \%$ CL. 


$$
\begin{aligned}
\delta g_{L}^{\text {new }}= & -\frac{m_{t}^{2}}{16 \pi^{2} v^{2}} \frac{\sin ^{2} \theta_{L}}{\left(1-\sin ^{2} \theta_{L}(1-x)\right)^{3}} \\
& \times\left(-1-\sin ^{4} \theta_{L}(1-x)^{3}+2 x\right. \\
& +\sin ^{2} \theta_{L}\left(2-5 x+2 x^{2}\right) \\
& \left.+\frac{\left(1-\sin ^{2} \theta_{L}\right) x(1+x) \ln x}{2(1-x)}\right) \\
& \simeq+\frac{m_{t}^{2}}{16 \pi^{2} v^{2}} \frac{\sin ^{2} \theta_{L}}{\left(1-\sin ^{2} \theta_{L}\right)}(x \ll 1),
\end{aligned}
$$

where $x$ is defined in Eq. (38). The constraint on $\delta g_{L}^{\text {new }}$ [31], which is derived from the constraint on $R_{b}$, is

$$
\delta g_{L}^{\text {new }}=(-5.8 \pm 8.6) \times 10^{-4} .
$$

Comparing Eqs. (50) and (51), we find the region $\left|\sin \theta_{L}\right|>0.52$ is excluded at $95 \% \mathrm{CL}$. This region is already excluded from the constraint on $S$ and $T$ parameters in Fig. 6. Hence, we conclude that the $Z b_{L} b_{L}$ constraint is not important in this model.

Before closing this section, we comment on the validity of our perturbative calculation. In the top sector, there are three parameters in the Lagrangian: $m, y_{t}$, and $y^{\prime}$. These three can be expressed by observables $m_{t}, m_{t^{\prime}}$, and $\theta_{L}$ as shown in Appendix A. In the limit $m_{t^{\prime}} \gg m_{t}$,

$$
y_{t} \simeq \frac{\sqrt{2}}{v} \frac{m_{t}}{\cos ^{2} \theta_{L}}, \quad y^{\prime} \simeq \frac{\sqrt{2}}{v} m_{t^{\prime}} \sin \theta_{L}, \quad M \simeq m_{t^{\prime}} \cos \theta_{L} .
$$

We have seen that the small $\theta_{L}$ region is allowed by the $S-T$ bound. Taking $\theta_{L} \ll 1$ limit, we get

$$
y_{t} \simeq \frac{\sqrt{2} m_{t}}{v} \simeq 1, \quad y^{\prime} \simeq \frac{m_{t^{\prime}}}{m_{t}} \sin \theta_{L}, \quad M \simeq m_{t^{\prime}} .
$$

We see that only $y^{\prime}$ provides a nontrivial constraint, especially in large $m_{t^{\prime}}$ region. In Fig. 6, we see that the allowed region of $\sin \theta_{L}$ is about less than 0.2 in large $m_{t^{\prime}}$ limit. Then, using Eq. (53), we see that

$$
y^{\prime} \lesssim 0.2 \frac{m_{t^{\prime}}}{m_{t}} .
$$

From this relation, we find that if we impose that $y^{\prime}$ should be lower than 4 to keep perturbativity, $m_{t^{\prime}}$ should be lighter than $3400 \mathrm{GeV}$. This upper bound on $m_{t^{\prime}}$ does not spoil our discussion in this paper. Thus, our perturbative calculation with $y^{\prime}$ is valid unless we take $m_{t^{\prime}}$ to be extremely large [32].

\section{CONCLUSIONS}

We have considered a possibility that the Higgs-like excesses observed at the LHC experiments are actually the signals of the dilaton associated with spontaneously broken scale invariance. We have constructed a minimal model of the dilaton which can be produced through the gluon fusion process at the LHC, and can decay into two photons. The effective coupling is obtained through the loop diagrams of a new vectorlike state $T$ that has the same gauge charges as the right-handed top quark. The $T$ field contributes to the Peskin-Takeuchi $S, T$ parameters in the electroweak precision tests. This contribution allows one to push the Higgs boson mass above the experimental constraint, $600 \mathrm{GeV}$, providing a consistent framework for the light dilaton plus a heavy Higgs scenario.

We find that the current experimental data allow distinct parameter regions where the excesses are either Higgs-like or dilatonlike. Once the excesses are confirmed with more statistics, it is possible to distinguish two scenarios.

\section{ACKNOWLEDGMENTS}

The authors thank the Yukawa Institute for Theoretical Physics at Kyoto University, where this work was initiated during the YITP workshop on "Beyond the Standard Model Physics (YITP-W-11-27)," March 19-23, 2012. The authors also acknowledge the participants of the workshop for very active discussions. We thank Masaya Ishino, Koji Nakamura, Yoshiko Ohno, Masaharu Tanabashi, and Junichi Tanaka for useful comments. This work is supported in part by the Grants-in-Aid for Scientific Research No. 23740165 (R. K.), No. 23104009, No. 20244028, No. 23740192 (K. O.), and No. 24340044 (J. S.) of JSPS.

Note added.-After completion of this manuscript, there appeared works treating a similar subject [34].

\section{APPENDIX A: MIXING OF TOP AND ITS PARTNER}

As said in the text, we have chosen the basis on which the mass mixing between top and its partner $\bar{T}_{L} u_{3 R}$ is rotated away,

$$
\left[\begin{array}{ll}
\overline{q_{3 L}} & \overline{T_{L}}
\end{array}\right]\left[\begin{array}{cc}
m & m^{\prime} \\
0 & M
\end{array}\right]\left[\begin{array}{c}
u_{3 R} \\
T_{R}
\end{array}\right]
$$

where $m=y_{t} v / \sqrt{2}$ and $m^{\prime}=y^{\prime} v / \sqrt{2}$. Switching to mass eigenstates

$$
\begin{aligned}
& {\left[\begin{array}{c}
q_{3 L} \\
T_{L}
\end{array}\right]=\left[\begin{array}{cc}
\cos \theta_{L} & \sin \theta_{L} \\
-\sin \theta_{L} & \cos \theta_{L}
\end{array}\right]\left[\begin{array}{c}
t_{L} \\
t_{L}^{\prime}
\end{array}\right],} \\
& {\left[\begin{array}{c}
u_{3 R} \\
T_{R}
\end{array}\right]=\left[\begin{array}{cc}
\cos \vartheta_{R} & \sin \vartheta_{R} \\
-\sin \vartheta_{R} & \cos \vartheta_{R}
\end{array}\right]\left[\begin{array}{c}
t_{R} \\
t_{R}^{\prime}
\end{array}\right],}
\end{aligned}
$$

we may diagonalize as

$$
\begin{aligned}
& {\left[\begin{array}{ll}
\bar{q}_{3 L} & \bar{T}_{L}
\end{array}\right]\left[\begin{array}{cc}
m & m^{\prime} \\
0 & M
\end{array}\right]\left[\begin{array}{c}
u_{3 R} \\
T_{R}
\end{array}\right]} \\
& \quad=\left[\begin{array}{ll}
\bar{t}_{L} & \bar{t}_{L}^{\prime}
\end{array}\right]\left[\begin{array}{cc}
m_{t} & 0 \\
0 & m_{t^{\prime}}
\end{array}\right]\left[\begin{array}{c}
t_{R} \\
t_{R}^{\prime}
\end{array}\right],
\end{aligned}
$$

where 


$$
\begin{aligned}
\tan \theta_{L} & =\frac{\sqrt{\left(M^{2}-m^{2}+m^{\prime 2}\right)^{2}+4 m^{\prime 2} m^{2}}-M^{2}+m^{2}+m^{\prime 2}}{2 m^{\prime} M} \\
& =\frac{m^{\prime}}{M}+O\left(M^{-3}\right), \\
\tan \vartheta_{R} & =\frac{\sqrt{\left(M^{2}-m^{2}+m^{\prime 2}\right)^{2}+4 m^{\prime 2} m^{2}}-M^{2}+m^{2}-m^{\prime 2}}{2 m^{\prime} m} \\
& =\frac{m^{\prime} m}{M^{2}}+O\left(M^{-4}\right),
\end{aligned}
$$

and the mass eigenvalues are

$$
\left\{\begin{array}{l}
m_{t}^{2} \\
m_{t^{\prime}}^{2}
\end{array}\right\}=\frac{M^{2}+m^{2}+m^{2} \mp \sqrt{\left(M^{2}+m^{2}+m^{\prime 2}\right)^{2}-4 m^{2} M^{2}}}{2} .
$$

For large $M$,

$$
\begin{aligned}
& m_{t}=\left(1-\frac{m^{\prime 2}}{2 M^{2}}\right) m+O\left(M^{-4}\right), \\
& m_{t^{\prime}}=M+\frac{m^{\prime 2}}{2 M}+O\left(M^{-3}\right) .
\end{aligned}
$$

Conversely, parameters in the Lagrangian can be written in terms of the observables,

$$
\begin{gathered}
M=\sqrt{m_{t}^{2} \sin ^{2} \theta_{L}+m_{t^{\prime}}^{2} \cos ^{2} \theta_{L}}, \\
y_{t}=\frac{\sqrt{2}}{v} \frac{m_{t} m_{t^{\prime}}}{\sqrt{m_{t}^{2} \sin ^{2} \theta_{L}+m_{t^{\prime}}^{2} \cos ^{2} \theta_{L}}}, \\
y^{\prime}=\frac{\sqrt{2}}{v} \frac{\left(m_{t^{\prime}}^{2}-m_{t}^{2}\right) \sin \theta_{L} \cos \theta_{L}}{\sqrt{m_{t}^{2} \sin ^{2} \theta_{L}+m_{t^{\prime}}^{2} \cos ^{2} \theta_{L}}} .
\end{gathered}
$$

Instead of our choice (A1), we may choose another basis where the Yukawa mixing $\bar{q}_{3 L} T_{R}$ is erased,

$$
\left[\begin{array}{ll}
\bar{q}_{3 L} & \bar{T}_{L}
\end{array}\right]\left[\begin{array}{cc}
m \cos \vartheta+m^{\prime} \sin \vartheta & 0 \\
M \sin \vartheta & M \cos \vartheta
\end{array}\right]\left[\begin{array}{c}
\tilde{u}_{3 R} \\
\tilde{T}_{R}
\end{array}\right],
$$

where

$$
\begin{aligned}
{\left[\begin{array}{c}
\tilde{u}_{3 R} \\
\tilde{T}_{R}
\end{array}\right] } & =\left[\begin{array}{cc}
\cos \vartheta & \sin \vartheta \\
-\sin \vartheta & \cos \vartheta
\end{array}\right]\left[\begin{array}{c}
u_{3 R} \\
T_{R}
\end{array}\right], \\
\tan \vartheta & =\frac{m^{\prime}}{m}=\frac{y^{\prime}}{y_{t}}
\end{aligned}
$$

Note that $\vartheta$ is not necessarily a small mixing angle and that the right-handed tops in this basis are related to the mass eigenstates by

$$
\left[\begin{array}{c}
t_{R} \\
t_{R}^{\prime}
\end{array}\right]=\left[\begin{array}{cc}
\cos \left(\vartheta+\vartheta_{R}\right) & -\sin \left(\vartheta+\vartheta_{R}\right) \\
\sin \left(\vartheta+\vartheta_{R}\right) & \cos \left(\vartheta+\vartheta_{R}\right)
\end{array}\right]\left[\begin{array}{c}
\tilde{u}_{3 R} \\
\tilde{T}_{R}
\end{array}\right]
$$

Although $\theta_{L}$ is more directly related to physical observables, one may trade it with $\vartheta+\vartheta_{R}$, which is the angle denoted by $\theta_{u}^{R}$ and is constrained in Ref. [33]. We find the following relation between $\theta_{L}$ and $\vartheta+\vartheta_{R}\left(=\theta_{u}^{R}\right)$ :

$$
\sin \theta_{L}=\frac{m_{t}}{\sqrt{m_{t}^{2} \sin ^{2} \theta_{u}^{R}+m_{t^{\prime}}^{2} \cos ^{2} \theta_{u}^{R}}} \sin \theta_{u}^{R}
$$

Using this relation, we find our result is compatible with the result given in Ref. [33].

\section{APPENDIX B: LINEAR REALIZATION POTENTIAL}

Though the precise form of the potential $\tilde{V}$ in Eq. (4) is irrelevant for the experimental consequences which are governed by the Higgs-dilaton mixing angle $\theta_{H}$ and the dilaton decay constant in units of the electroweak scale $\eta^{-1}=f / v$, let us write down a renormalizable linearized version of our potential just for completeness,

$$
\begin{aligned}
\tilde{V}(S, H)= & \frac{m_{S}^{2}}{2} S^{2}+\frac{\lambda_{S}}{4 !} S^{4}+\frac{\kappa}{2} S^{2}|H|^{2} \\
& +m_{H}^{2}|H|^{2}+\frac{\lambda_{H}}{2^{2}}|H|^{4} .
\end{aligned}
$$

The VEVs for the SM Higgs $\langle H\rangle=v / \sqrt{2}$ and for the singlet $\langle S\rangle=f$ are obtained as

$$
\left[\begin{array}{l}
f^{2} \\
v^{2}
\end{array}\right]=\frac{1}{\frac{\lambda_{S} \lambda_{H}}{6}-\kappa^{2}}\left[\begin{array}{cc}
\lambda_{H} & -2 \kappa \\
-2 \kappa & \frac{2 \lambda_{S}}{3}
\end{array}\right]\left[\begin{array}{c}
-m_{S}^{2} \\
-m_{H}^{2}
\end{array}\right]
$$

The mass eigenvalues are

$$
\left\{\begin{array}{l}
m_{s}^{2} \\
m_{h}^{2}
\end{array}\right\}=\frac{\left(\frac{\lambda_{S}}{3} f^{2}+\frac{\lambda_{H}}{2} v^{2}\right) \mp \sqrt{\left(\frac{\lambda_{S}}{3} f^{2}+\frac{\lambda_{H}}{2} v^{2}\right)^{2}-4\left(\frac{\lambda_{S} \lambda_{H}}{6}-\kappa^{2}\right) f^{2} v^{2}}}{2}
$$

with the Higgs-dilaton mixing (7) being

$$
\tan 2 \theta_{H}=\frac{2 \kappa f v}{\frac{\lambda_{S}}{3} f^{2}-\frac{\lambda_{H}}{2} v^{2}}
$$


Since we want $m_{s}^{2} / m_{h}^{2} \lesssim(125 \mathrm{GeV})^{2} /(600 \mathrm{GeV})^{2} \simeq 4 \% \ll 1$, we can write

$$
m_{s}^{2}=m_{\sigma}^{2}+O\left(\frac{m_{\sigma}^{4}}{M_{h}^{2}}\right), \quad m_{h}^{2}=M_{h}^{2}-m_{\sigma}^{2}+O\left(\frac{m_{\sigma}^{4}}{M_{h}^{2}}\right),
$$

with

$$
m_{\sigma}^{2}:=\left(\frac{\lambda_{S} \lambda_{H}}{6}-\kappa^{2}\right) \frac{f^{2} v^{2}}{M_{h}^{2}}, \quad M_{h}^{2}:=\frac{\lambda_{S}}{3} f^{2}+\frac{\lambda_{H}}{2} v^{2},
$$

where $\frac{\lambda_{S} \lambda_{H}}{6}-\kappa^{2}>0$ is required in order not to have a tachyon. Finally, we note that a tree-level vacuum stability condition for a large VEVs reads $\frac{2 \lambda_{S}}{3}-4 \kappa+\lambda_{H}>0$.

[1] ATLAS Collaboration, Phys. Lett. B 716, 1 (2012).

[2] CMS Collaboration, Phys. Lett. B 716, 30 (2012).

[3] S. Weinberg, Phys. Rev. D 13, 974 (1976); L. Susskind, Phys. Rev. D 20, 2619 (1979).

[4] C. Csaki, C. Grojean, H. Murayama, L. Pilo, and J. Terning, Phys. Rev. D 69, 055006 (2004).

[5] S. Matsuzaki and K. Yamawaki, Prog. Theor. Phys. 127, 209 (2012); Phys. Rev. D 85, 095020 (2012); 86, 035025 (2012); arXiv:1207.5911; D. Elander and M. Piai, arXiv:1208.0546; S. Matsuzaki and K. Yamawaki, arXiv:1209.2017.

[6] K. Yamawaki, M. Bando, and K.-i. Matumoto, Phys. Rev. Lett. 56, 1335 (1986); M. Bando, K.-i. Matumoto, and K. Yamawaki, Phys. Lett. B 178, 308 (1986).

[7] W. D. Goldberger, B. Grinstein, and W. Skiba, Phys. Rev. Lett. 100, 111802 (2008); J. Fan, W.D. Goldberger, A. Ross, and W. Skiba, Phys. Rev. D 79, 035017 (2009).

[8] R. Foot, A. Kobakhidze, and R. R. Volkas, Phys. Lett. B 655, 156 (2007); R. Foot, A. Kobakhidze, and K.L. McDonald, Eur. Phys. J. C 68, 421 (2010).

[9] K. Cheung and T.-C. Yuan, Phys. Rev. Lett. 108, 141602 (2012).

[10] B. Grzadkowski, J. F. Gunion, and M. Toharia, Phys. Lett. B 712, 70 (2012); Y. Tang, arXiv:1204.6145; H. Kubota and M. Nojiri, arXiv:1207.0621.

[11] M.E. Peskin and T. Takeuchi, Phys. Rev. D 46, 381 (1992); M.E. Peskin and T. Takeuchi, Phys. Rev. Lett. 65, 964 (1990).

[12] We also use $S$ and $T$ as the Peskin-Takeuchi parameters in Sec. IV. Notation might appear confusing, but it would easy to distinguish from the context.

[13] C. T. Hill, Phys. Lett. B 266, 419 (1991).

[14] B. A. Dobrescu and C. T. Hill, Phys. Rev. Lett. 81, 2634 (1998); R. S. Chivukula, B. A. Dobrescu, H. Georgi, and C. T. Hill, Phys. Rev. D 59, 075003 (1999); H.-J. He, C. T. Hill, and T. M.P. Tait, Phys. Rev. D 65, 055006 (2002).

[15] V. A. Miransky, M. Tanabashi, and K. Yamawaki, Phys. Lett. B 221, 177 (1989); Mod. Phys. Lett. A 04, 1043 (1989); W. A. Bardeen, C. T. Hill, and M. Lindner, Phys. Rev. D 41, 1647 (1990); Y. Nambu, Report No. EFI-89-08.

[16] A. Djouadi, Phys. Rep. 457, 1 (2008).

[17] P.P. Giardino, K. Kannike, M. Raidal, and A. Strumia, J. High Energy Phys. 06, (2012) 117.
[18] P.P. Giardino, K. Kannike, M. Raidal, and A. Strumia, arXiv: 1207.1347.

[19] D. Carmi, A. Falkowski, E. Kuflik, T. Volansky, and J. Zupan, J. High Energy Phys. 10 (2012) 196.

[20] ATLAS Collaboration, Report No. ATLAS-CONF-2012127.

[21] ATLAS Collaboration, Report No. ATLAS-CONF-2012091.

[22] CMS Collaboration, Report No. CMS PAS HIG-12-015.

[23] ATLAS Collaboration, Report No. ATLAS-CONF-2012092.

[24] CMS Collaboration, Report No. CMS PAS HIG-12-016.

[25] ATLAS Collaboration, Report No. ATLAS-CONF-2012098.

[26] CMS Collaboration, Report No. CMS PAS HIG-12-017.

[27] ALEPH Collaboration, CDF Collaboration, D0 Collaboration, DELPHI Collaboration, L3 Collaboration, OPAL Collaboration, SLD Collaboration, LEP Electroweak Working Group, Tevatron Electroweak Working Group, and SLD Electroweak and Heavy Flavour Groups, arXiv:1012.2367.

[28] CMS Collaboration, Phys. Rev. Lett. 107, 271802 (2011); CMS Collaboration, Phys. Lett. B 716, 103 (2012); CMS Collaboration, Report No. CMS PAS EXO-11-099.

[29] V. M. Abazov et al. (D0 Collaboration), Phys. Rev. D 85, 091104 (2012).

[30] J. Beringer et al. (Particle Data Group Collaboration), Phys. Rev. D 86, 010001 (2012).

[31] T. Abe, R. S. Chivukula, N.D. Christensen, K. Hsieh, S. Matsuzaki, E.H. Simmons, and M. Tanabashi, Phys. Rev. D 79, 075016 (2009).

[32] A tighter upper bound would be imposed, roughly $M \lesssim$ $1 \mathrm{TeV}$ depending on $\eta$, if one requires perturbativity for the coupling $\frac{M}{f} S T T$, though it is irrelevant for the computations in this paper.

[33] G. Cacciapaglia, A. Deandrea, L. Panizzi, N. Gaur, D. Harada, and Y. Okada, J. High Energy Phys. 03 (2012) 070.

[34] Z. Chacko and R. K. Mishra, arXiv:1209.3022; Z. Chacko, R. Franceschini, and R. K. Mishra, arXiv:1209.3259; B. Bellazzini, C. Csaki, J. Hubisz, J. Serra, and J. Terning, arXiv:1209.3299. 